\title{
THE DENSITY PROCESS OF THE MINIMAL ENTROPY MARTINGALE MEASURE IN A STOCHASTIC VOLATILITY MARKET. A PDE APPROACH.
}

\author{
RODWELL KUFAKUNESU
}

\begin{abstract}
In a stochastic volatility market the Radon-Nikodym density of the minimal entropy martingale measure can be expressed in terms of the solution of a semilinear PDE. This fact has been explored and illustrated for the time-homogeneous case in a recent paper by Benth and Karlsen [3]. However, there are some cases which time-dependent parameters are required such as when it comes to calibration. This paper generalizes their model to the time-inhomogeneous case.
\end{abstract}

Mathematics Subject Classification (2000): 93E20; 35K55; 91G80.

\section{INTRODUCTION}

Let $(\Omega, \mathcal{F}, P)$ be a complete probability space equipped with a filtration $\left\{\mathcal{F}_{t}\right\}_{t \in[0, T]}$ satisfying the usual conditions, where $T<\infty$ is the time horizon. Let $B_{t}$ and $W_{t}$ be standard Brownian motions defined on a filtered probability space, and suppose the risky asset $S$ evolves according to the following general stochastic volatility model:

$$
d S_{t}=\mu\left(t, Y_{t}\right) S_{t} d t+\sigma\left(t, Y_{t}\right) S_{t} d B_{t}, \quad S(0)>0,
$$

where $Y_{t}$ is a stochastic volatility process driven by the process

$$
d Y_{t}=\alpha\left(t, Y_{t}\right) d t+\beta\left(t, Y_{t}\right) d B_{t}+\delta\left(t, Y_{t}\right) d W_{t}, \quad Y(0)>0 .
$$

We assume that the time-dependent parameter functions $\mu:[0, \infty) \times \mathbb{R} \rightarrow \mathbb{R}, \sigma:[0, \infty) \times$ $\mathbb{R} \rightarrow \mathbb{R}, \alpha:[0, \infty) \times \mathbb{R} \rightarrow \mathbb{R}_{+}, \beta:[0, \infty) \times \mathbb{R} \rightarrow \mathbb{R}_{+}$, and $\delta:[0, \infty) \times \mathbb{R} \rightarrow \mathbb{R}_{+}$, are Borel measurable functions on $\mathbb{R}$ and continuous on $[0, \infty) \times \mathbb{R}$. Furthermore, we assume that the parameters $\alpha, \beta$ and $\delta$ are defined in such a way that $\sigma\left(t, Y_{t}\right):=Y_{t}$ is nonnegative, such that a unique strong solution of the above stochastic differential equations (1.1)-(1.2) exists. (More conditions on particular cases of stochastic volatility models are discussed in Section 3 of this paper). Without loss of generality, we suppose that the rate of return from a risk-free investment is zero.

In an incomplete market a claim cannot be perfectly replicated as in the complete one and there exists a continuum of equivalent martingale measures $Q$ and, correspondingly, arbitrage free prices. The question is how to select this measure and fix the price of a contingent claim. Several approaches have been suggested in literature. We refer to a

Key words and phrases. Utility optimisation, stochastic volatility, incomplete market, minimal entropy martingale measure, Hamilton-Jacobi-Bellman equation. 
discussion in Pham [12] for a general overview, of the super-hedging, mean variance hedging and shortfall risk minimization.

Herein, as in Benth and Karlsen [3] we are interested in the minimal entropy martingale measure (MEMM) and the utility indifference pricing approach, which was introduced by Hodges and Neuberger [14] (see also other references given in Benth and Karlsen [3]). In utility indifference pricing, one considers the difference between maximum utility from final wealth when there is no contingent claim liability and when there is such a liability, and then define the price as the unique cash increment which offsets the difference.

Our stochastic volatility market given by equations (1.1)-(1.2) is incomplete as soon as the parameter $\delta(t, y) \neq 0$ for all $t>0$, and we are interested in using the MEMM for pricing contingent claims. A martingale measure is a probability measure $Q$ on a measurable space $(\Omega, \mathcal{F})$ such that $Q \ll P$ and $S$ is a local $Q$-martingale. We denote by $\mathcal{M}$ the set of martingale measures and by $\mathcal{M}_{e}$ the set of martingale measures that are equivalent to $P$. Let $Q$ be a probability measure $(\Omega, \mathcal{F})$. The relative entropy, or KullbackLeibler distance, $H(Q, P)$ of $Q$ with respect to $P$ is defined as in Frittelli [8] and Benth and Karlsen [3]:

$$
H(Q, P)= \begin{cases}\mathbb{E}\left[\frac{d Q}{d P} \ln \left(\frac{d Q}{d P}\right)\right], & Q \ll P \\ +\infty, & \text { otherwise }\end{cases}
$$

where $\mathbb{E}$ is the expectation operator under $P$. We look for a probability measure $Q_{M E}$ that minimizes the relative entropy with respect to $P$ in the class $\mathcal{M}$. We call $Q_{M E}$ a MEMM. More precisely, we call $Q_{M E}$ a MEMM if

$$
H\left(Q_{M E}, P\right)=\min _{Q \in \mathcal{M}} H(Q, P) .
$$

In Frittelli [8], it is proved that if there exists a $Q \in \mathcal{M} e$ with $H(Q, P)<\infty$, then $Q_{\mathcal{M E}}$ exists, is unique, and is equivalent to $P$ (i.e., $Q_{M E} \in Q_{\mathcal{M e}}$ ).

Recently, Rheinländer [16] presented a martingale duality method for finding the MEMM in a general continuous semimartingale model, and derived sufficient conditions verifying that a candidate measure is the MEMM. He illustrated his method on the Stein-Stein stochastic volatility model with time-independent parameters, where he calculated explicitly the density of the MEMM by solving an integral equation resulting from a (non-trivial) martingale representation theorem.

One objective of this paper is to show that one can determine the MEMM via the solution of a semilinear partial differential equation (PDE) as in Benth and Karlsen [3], extending it to time dependence on some parameters. For the stochastic volatility models with at most linearly growing drift and additive noise correlated in a time dependent manner with the asset dynamics, we show that a unique classical solution to the semilinear PDE exists, thereby paving way for a simpler approach to derive explicitly the density of the MEMM with time-dependent parameters, thereby generalizing the results obtained in Benth and Karlsen [3].

We illustrate our PDE approach with explicit calculations of the MEMM $Q_{M E}$ for the Stein-Stein model and Heston stochastic volatility models, as in Benth and Karlsen [3]. 
In an incomplete market driven by continuous semimartingales, the work by Mania, Santacrose and Tevzadze [17] proves that the density of the MEMM can be expressed in terms of a value process that is a unique solution to a semimartingale backward stochastic differential equation. Our PDE approach goes as follows:- Suppose there exists a unique classical solution $v=v(t, y)$ of the semilinear PDE

$$
-v_{t}-\frac{1}{2} a^{2}(t, y) v_{y y}+F\left(t, y, v_{y}\right)=0, \quad(t, y) \in[0, T] \times \mathbb{R},
$$

with terminal condition

$$
v(T, y)=0, \quad y \in \mathbb{R},
$$

where $a^{2}(t, y)=\beta^{2}(t, y)+\delta^{2}(t, y)$ and the nonlinear function

$F:[0, T) \times \mathbb{R} \times \mathbb{R} \rightarrow \mathbb{R}$ is defined as

$$
F(t, y, p)=\frac{1}{2} \delta^{2}(t, y) p^{2}-\left[\alpha(t, y)-\frac{\mu(t, y) \beta(t, y)}{\sigma(t, y)}\right] p-\frac{1}{2} \frac{\mu^{2}(t, y)}{\sigma^{2}(t, y)}
$$

The term "classical solution" means that $v(t, y)$ is once continuously differentiable in $t$ and twice continuously differentiable in $y$ for $(t, y) \in[0, T) \times \mathbb{R}$, and continuous in $t$ and $y$ for $(t, y) \in[0, T) \times \mathbb{R}$, that is,

$$
v \in C^{1,2}([0, T) \times \mathbb{R}) \cap C([0, T) \times \mathbb{R})
$$

and $v$ satisfies equations (1.3) and (1.4) in the usual pointwise sense. Furthermore, suppose that

$$
\int_{0}^{T} \frac{\mu^{2}\left(t, Y_{t}\right)}{\sigma^{2}\left(t, Y_{t}\right)} d t<\infty, \quad \int_{0}^{T} \delta^{2}\left(t, Y_{t}\right) v_{y}^{2}\left(t, Y_{t}\right) d t<\infty, \quad P-a . s .
$$

for all $t>0$. Define for $0 \leq t \leq T$ the stochastic process $Z_{t}$ by

$$
\begin{aligned}
Z_{t}=\exp & \left(-\int_{0}^{t} \frac{\mu\left(s, Y_{s}\right)}{\sigma\left(s, Y_{s}\right)} d B_{s}-\int_{0}^{t} \delta\left(s, Y_{s}\right) v_{y}\left(s, Y_{s}\right) d W_{s}\right. \\
& \left.-\frac{1}{2} \int_{0}^{t}\left(\frac{\mu^{2}\left(t, Y_{s}\right)}{\sigma^{2}\left(t, Y_{s}\right)}+\delta^{2}\left(s, Y_{s}\right) v_{y}^{2}\left(s, Y_{s}\right)\right) d s\right),
\end{aligned}
$$

which is well-defined by the assumptions in equation (1.5). Another purpose of this paper is to verify under natural additional assumptions on $v$ and the time-dependent parameters of the diffusion dynamics (1.1) and (1.2) that $Z_{T}$ is the Radon-Nikodym derivative of the MEMM $Q_{M E}$. The argument applies a verification result in Rheinländer [16]. Another main purpose of this paper is to extend results obtained in Benth and Karlsen [3] to incorporate time-dependent parameters in giving sufficient conditions and a proof for the well-posedness of equations (1.3) and (1.4) in the framework of classical solutions. We shall follow the approach used in Benth and Karlsen [3], where the proof is based on stochastic control theory.

We derive the MEMM with respect to the filtration $\mathcal{F}$ (generated by the Brownian motions $B$ and $W$ ), and not generated by the asset price process $\mathcal{F}^{S}$. For many volatility models, these filtrations coincide, in particular, when volatility is positive. We shall make 
the assumption that the investor has complete information, that is, we derive the MEMM with respect to $\mathcal{F}$ and also in the case $\mathcal{F} \neq \mathcal{F}^{S}$. We refer to Rheinländer [16] and Hobson [10] for an analysis of the Stein-Stein model and MEMM with respect to different information sets.

The structure of the paper is much like parts of Benth and Karlsen [3], we often refer to passages therein. In Section 2, we derive the semilinear PDE formally. In Section 3, under certain conditions $A$ and $B$ we prove that $Z_{T}$ is the density of the MEMM $Q_{M E}$. In Section 4 , we discuss the well-posedness of semilinear PDE terminal value problems like (1.3)-(1.4) coming from stochastic volatility models with additive noise and at most a linearly growing drift. We use this to identify the density of the MEMM $Q_{M E}$ for this class of volatility models (verifying the Stein-Stein model as a special case). Finally, Section 5 is devoted to verify the conditions needed in Section 3 for the Heston volatility model, which does not have a linearly growing drift, that is, it does not fit into the framework in Section 4.

1.1. Formal Derivation of the semilinear PDE. We want to determine the utility indifference price (see Benth and Karlsen [3] and references therein) of a European type contingent claim in the stochastic volatility market with time dependent parameters equations (1.1) and (1.2). The utility indifference price will be derived by solving two utility maximization problems. We shall use the dynamic programming (or Bellman) method to solve the two stochastic control problems.

We assume that the market to invest in consists of a risky asset $S_{t}$ given by equations (1.1) and (1.2) or a risk-free bond yielding a sure rate of return 0, that is, a bond with dynamics $R_{t}=1$. If the investor allocates the amount $\pi_{t}$ of her wealth at time $t$ in the risky asset, it follows from the self-financing hypothesis that:

$$
d X_{t}=\pi_{t} \mu\left(t, Y_{t}\right) d t+\sigma\left(t, Y_{t}\right) \pi_{t} d B_{t} .
$$

The control $\pi$ is called admissible if it is an adapted stochastic process for which there exists a wealth process $X_{t}{ }^{\pi}$ satisfying the stochastic differential equation (1.7) and such that $\mathbb{E}\left[\int_{0}^{T} \pi_{t}^{2} \sigma^{2}\left(t, Y_{t}\right) d t\right]<\infty$, for all $t>0$. We denote a set of all such controls by $\mathcal{A}_{t}$, where the subscript $t$ indicates that we start the wealth dynamics at time $t$. We assume that the investor has full information about the volatility since the controls are assumed adapted to $\mathcal{F}_{t}$ and not only to the filtration generated by the asset price process $\mathcal{F}_{t}{ }^{s}$. For many volatility models these filtrations coincide, in particular when volatility is positive.

Considering Markov controls, this entails that the investor will allocate the amount $\pi \equiv \pi(t, x, y)$ into the risky asset when the wealth $X_{t}=x$ and volatility $Y_{t}=y$. Her goal is to find the investment strategy that maximizes her final utility. We will also consider the same investor who first issues a claim and then maximizes her final utility. The claim is of the European type and we assume a claim which has a pay-off at time $T$, given by $g\left(S_{T}\right)$, where $g$ is a bounded and measurable function. We shall only deal with Markovian claims. Consider an exponential utility function $U:[0, T] \rightarrow \mathbb{R}$, given by

$$
U(x)=1-\exp (-\gamma x)
$$


where $\gamma>0$ is the risk-aversion parameter. With utility function of exponential type for which the index of risk is independent of the investor's wealth, we are able to separate the value function's dependence of wealth and volatility. This will lead to a price of the claim that is independent of the investor's initial wealth. The resulting value in this case is:

$$
V^{0}(t, x, y)=\sup _{\pi \in \mathcal{A}_{t}} \mathbb{E}\left[1-\exp \left(-\gamma X_{T}\right) \mid X_{t}=x, Y_{t}=y\right] .
$$

In the second stochastic control problem we suppose that a claim has been issued. The final wealth then becomes $X_{T}-g\left(S_{T}\right)$ and the value function is therefore

$$
V(t, x, y, s)=\sup _{\pi \in \mathcal{A}_{t}} \mathbb{E}\left[1-\exp \left(-\gamma\left(X_{T}-g\left(S_{T}\right)\right)\right) \mid X_{t}=x, Y_{t}=y, S_{t}=s\right] .
$$

By selling the claim at time $t$ when a stock price is $s$ and the volatility is given by $y$, the issuer will charge a premium $\wedge_{t}{ }^{(\gamma)}(t, y, s)$ and optimally invest in the market. The premium is fixed such that the investor is indifferent between investing at her own account or issuing the claim and then investing. Hence $\wedge_{t}{ }^{(\gamma)}(t, y, s)$ should satisfy:

$$
V^{0}(t, x, y, s)=V\left(t, x+\wedge_{t}{ }^{(\gamma)}(t, y, s), y, s\right) .
$$

The utility indifference price is defined as the unique solution $\wedge_{t}{ }^{(\gamma)}(t, y, s)$ of equation (1.10). By formally solving the two stochastic control problems (1.8) and (1.9) by the dynamic programming method, we will be able to determine a semilinear PDE satisfied by $\wedge_{t}{ }^{(\gamma)}(t, y, s)$. Now, we formulate the Hamilton-Jacobi-Bellman (HJB) equation for the value function (1.8) without a claim issued. It reads:

$$
\begin{array}{r}
V_{t}^{0}+\max _{\pi \in \mathbb{R}}\left[\pi \mu(t, y) V_{x}^{0}+\frac{1}{2} \sigma^{2}(t, y) \pi^{2} V_{x x}^{0^{2}}+\beta(t, y) \sigma(t, y) \pi V_{x y}^{0}\right] \\
+\mathcal{L}_{y} V^{0}=0, \quad(t, x, y) \in[0, T) \times \mathbb{R} \times \mathbb{R},
\end{array}
$$

where

$$
\mathcal{L}_{y} V^{0}=\alpha(t, y) V_{y}^{0}+\frac{1}{2}\left\{\beta^{2}(t, y)+\delta^{2}(t, y)\right\} V_{y y}^{0}
$$

with terminal data

$$
V^{0}(T, x, y)=1-\exp (-\gamma x),(x, y) \in \mathbb{R} \times \mathbb{R} .
$$

We formulate the HJB for the value function (1.11) when the investor has issued a claim with payoff function $g(s)$ at time $\mathrm{T}$. It reads:

$$
\begin{aligned}
V_{t} & +\max _{\pi \in \mathbb{R}}\left[\pi \mu(t, y) V_{x}+\frac{1}{2} \sigma^{2}(t, y) \pi^{2} V_{x x}+\beta(t, y) \sigma(t, y) \pi V_{x y}+\sigma^{2}(t, y) \pi s V_{x s}\right] \\
& +\mathcal{L}_{y} V+\mathcal{L}_{s} V+\sigma(t, y) \beta(t, y) s V_{y s}=0, \quad(t, x, y, s) \in[0, T) \times \mathbb{R} \times \mathbb{R} \times \mathbb{R}_{+} .
\end{aligned}
$$

where $\mathcal{L}_{y}$ is defined in equation (1.11) and

$$
\mathcal{L}_{s} V=\mu(t, y) s V_{s}+\frac{1}{2} \sigma^{2}(t, y) s^{2} V_{s s},
$$


with terminal data

$$
V(T, x, y, s)=\exp (-\gamma(x-g(s))) .
$$

From equation (1.11) we find out that the first order condition for an optimal investment strategy is

$$
\mu(t, y) V_{x}^{0}+\frac{1}{2} \sigma^{2}(t, y) \pi V_{x x}^{0}+\beta(t, y) \sigma(t, y) V_{x y}^{0}=0 .
$$

Solving for $\pi^{*}$ we have

$$
\pi^{*}=-\frac{\mu(t, y) V_{x}^{0}+\beta(t, y) \sigma(t, y) V_{x y}^{0}}{\sigma^{2}(t, y) V_{x x}^{0}} .
$$

Inserting $\pi^{*}$ into the HJB equation (1.11) yields the following non-linear PDE

$$
\begin{aligned}
V_{t}^{0} & +\left[-\frac{\mu(t, y) V_{x}^{0}+\beta(t, y) \sigma(t, y) V_{x y}^{0}}{\sigma^{2}(t, y) V_{x x}^{0}}\right] \mu(t, y) V_{x}^{0} \\
& +\frac{1}{2} \sigma^{2}(t, y)\left[-\frac{\mu(t, y) V_{x}^{0}+\beta(t, y) \sigma(t, y) V_{x y}^{0}}{\sigma^{2}(t, y) V_{x x}^{0}{ }^{2}}\right]^{2} V_{x x}^{0} \\
& +\beta(t, y) \sigma(t, y)\left[-\frac{\mu(t, y) V_{x}^{0}+\beta(t, y) \sigma(t, y) V_{x y}^{0}}{\sigma^{2}(t, y) V_{x x}^{0}}\right] V_{x y}^{0} \\
& +\mathcal{L}_{y} V^{0}=0,(t, x, y) \in[0, T) \times \mathbb{R} \times \mathbb{R} .
\end{aligned}
$$

We reduce the state space by one dimension, by making

$$
V^{0}(t, x, y)=1-\exp (-\gamma x-v(t, y)) .
$$

This transform simplifies the non-linearities in equation (1.15) considerably. Differentiating equation (1.16) we have:

$$
\begin{array}{r}
v_{t}+\frac{\mu^{2}(t, y)}{2 \sigma^{2}(t, y)}-\frac{\beta(t, y) \mu(t, y)}{\sigma(t, y)} v_{y}-\frac{1}{2} \delta^{2}(t, y) v_{y}^{2} \\
+\mathcal{L}_{y} v=0, \quad(t, y) \in[0, T) \times \mathbb{R},
\end{array}
$$

with terminal data

$$
v(T, y)=0, \quad y \in \mathbb{R} .
$$

Here the idea of using a logarithmic transformation to reduce the non-linearities in the HJB equation was used in Benth and Karlsen [3]. From equations (1.13), (1.14) and the first order condition, we can derive the following expression for the optimal investment strategy $\pi^{*}$ :

$$
\pi^{*}=-\frac{\mu(t, y) V_{x}+\beta(t, y) \sigma(t, y) V_{x y}+\sigma^{2}(t, y) s V_{x s}}{\sigma^{2}(t, y) V_{x x}} .
$$

Inserting $\pi^{*}$ into the HJB equation (1.13) yields as before the non-linear PDE becomes:

$$
V_{t}-\frac{\mu^{2}(t, y) V_{x}^{2}}{2 \sigma^{2}(t, y) V_{x x}}-\frac{\beta^{2}(t, y) V_{x y}^{2}}{2 V_{x x}}-\frac{\sigma^{2}(t, y) s V_{x s}^{2}}{2 V_{x x}}
$$




$$
\begin{aligned}
& -\frac{\mu(t, y) \beta(t, y) V_{x} V_{x y}}{\sigma(t, y) V_{x x}}-\frac{\mu(t, y) s V_{x} V_{x s}}{V_{x x}}-\frac{\sigma(t, y) \beta(t, y) s V_{x y} V_{x s}}{V_{x x}} \\
& +\mathcal{L}_{y} V+\mathcal{L}_{s} V+\sigma(t, y) \beta(t, y) s V_{y s}=0,(t, x, y, s) \in[0, T) \times \mathbb{R} \times \mathbb{R} \times \mathbb{R}_{+} .
\end{aligned}
$$

We now make the ansatz,

$$
V(t, x, y, s)=1-\exp (-\gamma x+\gamma f(t, y, s)-v(t, y)),
$$

for some function $f(t, y, s)$ to be determined and with $v(t, y)$ solving equation (1.17).

With this representation the utility indifference price for the claim will be given by $f(t, y, s)$. By the representations (1.16) and (1.19) and the definition of the utility indifference price (1.10), we have

$$
1-\exp \left(-\gamma\left(x+\wedge^{(\gamma)}(t, y, s)\right)+\gamma f(t, y, s)-v(t, y)\right)=1-\exp (-\gamma x-v(t, y)),
$$

which implies that

$$
f(t, y, s)=\wedge^{(\gamma)}(t, y, s) .
$$

Differentiating equation (1.19) and deriving a semilinear PDE for $\wedge^{(\gamma)}$, that is, for the option price for a general risk-aversion parameter, we have:

$$
\begin{array}{r}
-\wedge_{t}^{(\gamma)}-\frac{1}{2} \sigma^{2}(t, y) s^{2} \wedge_{s s}^{(\gamma)}-\sigma(t, y) \beta(t, y) s \wedge_{y s}^{(\gamma)}+\left[\delta^{2}(t, y) v_{y}-\frac{\mu(t, y) \beta(t, y)}{\sigma(t, y)}\right] \wedge_{y}^{(\gamma)} \\
-\frac{1}{2} \gamma \delta^{2}(t, y)\left(\wedge_{y}^{(\gamma)}\right)^{2}-\mathcal{L}_{y} \wedge^{(\gamma)}=0, \quad(t, y, s) \in[0, T) \times \mathbb{R} \times \mathbb{R}_{+} .
\end{array}
$$

Since

$$
V(T, x, y, s)=1-\exp (-\gamma(x-g(s)))
$$

holds, $\wedge^{(\gamma)}$ obeys the terminal condition:

$$
\wedge^{(\gamma)}(T, y, s)=g(s), \quad(y, s) \in \mathbb{R} \times \mathbb{R}_{+} .
$$

When $\delta=0$, we have the complete case to equation (1.20). The PDE (1.20) then becomes:

$$
\begin{array}{r}
\wedge_{t}^{(\gamma)}+\frac{1}{2} \sigma^{2}(t, y) s^{2} \wedge_{s s}^{(\gamma)}+\left[\alpha(t, y)-\frac{\mu(t, y) \beta(t, y)}{\sigma(t, y)}\right] \wedge_{y}^{(\gamma)} \\
+\frac{1}{2} \beta^{2}(t, y) \wedge_{y y}^{(\gamma)}+\sigma(t, y) \beta(t, y) s \wedge_{y s}^{(\gamma)}=0
\end{array}
$$

Introducing the Girsanov transformation of $B_{t}$ given by

$$
d B_{t}=-\frac{\mu\left(t, Y_{t}\right)}{\sigma\left(t, Y_{t}\right)} d t+d \tilde{B}_{t}
$$

where $\tilde{B}_{t}$ is a Brownian motion under $Q$ (at least when the Novikov for $\frac{\mu\left(t, Y_{t}\right)}{\sigma\left(t, Y_{t}\right)}$ holds). The unique arbitrage free price of the contingent claim is

$$
\mathbb{E}_{Q}\left[g\left(S_{T}\right) \mid Y_{t}=y, S_{t}=s\right],
$$

and this expression solves (1.21) and (1.22) using the notions of the Feynman-Kac formula (see Øksendal [11], for details of this formula). Thus, in the complete market case, the 
arbitrage free price coincides with the utility indifference price.

We suppose that the zero risk aversion limit:

$$
\wedge(t, y, s):=\lim _{\gamma \rightarrow 0} \wedge^{(\gamma)}(t, y, s),
$$

exists and that $\wedge_{y}^{(\gamma)}(t, y, s)$ remains uniformly bounded as $\gamma \rightarrow 0$ for each fixed $(t, y, s)$. Then, from equation (1.21) $\wedge$ satisfies the linear Black-Scholes type PDE problem

$$
\left\{\begin{array}{l}
\wedge_{t}+\frac{1}{2} \sigma^{2}(t, y) s^{2} \wedge_{s s}+\mathcal{L}_{y} \wedge-\left(\delta^{2}(t, y) v_{y}+\frac{\mu(t, y) \beta(t, y)}{\sigma(t, y)}\right) \wedge_{y} \\
+\sigma(t, y) \beta(t, y) s \wedge_{y s}=0, \quad(t, y, s) \in[0, T) \times \mathbb{R} \times \mathbb{R}_{+} \\
\wedge(T, y, s)=g(s), \quad(y, s) \in \mathbb{R} \times \mathbb{R}_{+} .
\end{array}\right.
$$

The Feynman-Kac formula yields the following representation for $\wedge$

$$
\mathbb{E}\left[g\left(\tilde{S}_{T}\right) \mid \tilde{Y}_{t}=y, \tilde{S}_{t}=s\right],
$$

where the stochastic processes $\tilde{S}$ and $\tilde{Y}$ are given by

$$
\begin{aligned}
d \tilde{S}_{t}= & \sigma\left(t, \tilde{Y}_{t}\right) \tilde{S}_{t} d \tilde{B}_{t}, \\
d \tilde{Y}_{t}= & \left(\alpha\left(t, \tilde{Y}_{t}\right)-\delta^{2}\left(t, \tilde{Y}_{t}\right) v_{y}\left(t, \tilde{Y}_{t}\right)-\frac{\mu\left(t, \tilde{Y}_{t}\right) \beta\left(t, \tilde{Y}_{t}\right.}{\sigma\left(t, \tilde{Y}_{t}\right)}\right) d t \\
& +\beta\left(t, \tilde{Y}_{t}\right) d \tilde{B}_{t}+\delta\left(t, \tilde{Y}_{t}\right) d \tilde{W}_{t} .
\end{aligned}
$$

Formally, we rewrite equation (1.24) in terms of the original processes under the equivalent martingale measure. Introduce the Girsanov transformations:

$$
\begin{aligned}
d B_{t} & =d \tilde{B}_{t}-\frac{\mu\left(t, Y_{t}\right)}{\sigma\left(t, Y_{t}\right)} d t, \\
d W_{t} & =d \tilde{W}_{t}-\delta\left(t, Y_{t}\right) v_{y}\left(t, Y_{t}\right) d t,
\end{aligned}
$$

where $\tilde{B}$, and $\tilde{W}$ are two independent Brownian motions under the equivalent martingale measure $Q$, whose Radon-Nikodym derivative is $\frac{d Q}{d P}=Z_{T}$ with

$$
\begin{aligned}
Z_{T}= & \exp \left(-\int_{0}^{T} \frac{\mu\left(t, Y_{t}\right)}{\sigma\left(t, Y_{t}\right)} d B_{t}-\int_{0}^{T} \delta\left(t, Y_{t}\right) v_{y}\left(t, Y_{t}\right) d W_{t}\right. \\
& \left.-\frac{1}{2} \int_{0}^{T}\left(\frac{\mu^{2}\left(t, Y_{t}\right)}{\sigma^{2}\left(t, Y_{t}\right)}+\delta^{2}\left(t, Y_{t}\right) v_{y}^{2}\left(t, Y_{t}\right)\right) d t\right) .
\end{aligned}
$$

Then equation (1.24) takes the form:

$$
\wedge(t, y, s)=\mathbb{E}_{Q}\left[g\left(S_{T}\right) \mid Y_{t}=y, S_{t}=s\right] .
$$

This Girsanov transform is valid as long as the process $Z$ is a martingale. 
We now prove (under certain conditions) that $Q$ defined by equation (1.25) coincides with the MEMM $Q_{M E}$ and $\wedge$ defined in equation (1.26) is hence just the arbitrage free price under $Q_{M E}$ (the so called minimal entropy price). From general duality theory (see Benth and Karlsen [3] and references therein) without reference to PDEs, it is known that the zero risk aversion asymptotic of the utility indifference price with exponential utility coincides with the minimal entropy price. So, we propose $Z_{T}$ given in equation (1.25) as the candidate density for the minimal entropy measure.

\section{IDENTIFICATION OF THE MEMM}

In this section we want to prove that $Z_{T}$ given by equation (1.25) is the density of the MEMM $Q_{M E}$. To this end we need to verify that $Z_{T}$ is a martingale (not only a local martingale) defining a probability measure with finite relative entropy. We do this by verifying certain conditions given in Rheinländer [16] and in Benth and Karlsen [3], with some modification. Firstly, we show that $S Z$ is a local $P$-martingale (thus $S$ is a local $Q$ martingale), where $S$ solves equation (1.1). Secondly, applying Itô's formula on the product $S Z$ we have:

$$
\begin{aligned}
d(S Z)_{t}= & S_{t} d Z_{t}+Z_{t} d S_{t}+d[S Z]_{t} \\
= & \left(\sigma\left(t, Y_{t}\right)-\frac{\mu\left(t, Y_{t}\right)}{\sigma\left(t, Y_{t}\right)}\right)(S Z)_{t} d B_{t} \\
& -\delta\left(t, Y_{t}\right) v_{y}\left(t, Y_{t}\right)(S Z)_{t} d W_{t} .
\end{aligned}
$$

If $Z$ is a martingale, and not merely a local martingale, $Z_{T}$ will be the density of a martingale measure. Following the notation in Rheinländer [16], we introduce the following conditions:

$$
\begin{aligned}
K_{t} & =\int_{0}^{t} \frac{\mu^{2}\left(u, Y_{u}\right)}{\sigma^{2}\left(u, Y_{u}\right)} d u, \\
L_{t} & =-\int_{0}^{t} \delta\left(u, Y_{u}\right) v_{y}\left(u, Y_{u}\right) d W_{u},
\end{aligned}
$$

and its quadratic variation denoted by $[L]_{t}$ is given as

$$
[L]_{t}=\int_{0}^{t} \delta^{2}\left(u, Y_{u}\right) v_{y}^{2}\left(u, Y_{u}\right) d u .
$$

Define for each natural number $n$ the stopping time

$$
\tau_{n}=\inf \left\{t>0 \mid \max \left(K_{t},[L]_{t}\right) \geq n\right\}, \quad T_{n}=\min \left(\tau_{n}, T\right) .
$$

Set

$$
Z_{t}^{n}=Z_{t \wedge T_{n}}
$$

Novikov's criterion now implies that $Z_{T}^{n}$ is the density of a probability measure $Q_{n}$. From Rheinländer [16] we have the following:

Theorem 2.1. [16]. The following assertions are equivalent:

(1) $\sup _{n} \mathbb{E}_{Q_{n}}\left[K_{T_{n}}+[L]_{T_{n}}\right]<\infty$, 
(2) $\sup _{n} H\left(Q_{n}, P\right)<\infty$,

(3) $Z_{T}$ is the density of a probability measure $Q$ with $H(Q, P)<\infty$.

We introduce the following condition:

Condition A. Let the functions $\mu(t, y), \sigma(t, y), \bar{\beta}(t, y)$, and $v(t, y)$ be such that assertion (1) or equivalently assertion (2) in Theorem 2.1 holds.

Under Condition $A$ we ensured that $Z_{T}$ is the density of a probability measure $Q$ with finite relative entropy, so that $Q$ is in fact a martingale measure with finite relative entropy. Our next task is to find conditions such that this measure has minimal entropy. We will first rewrite the expression for $Z_{T}$ as

$$
\exp \left(c+\int_{0}^{T} \eta_{t} d S_{t}\right)
$$

for a constant $c$ and some adapted process $\eta_{t}$, and then we will identify $Z_{T}$ as the density of MEMM $Q_{M E}$ by verifying the condition in the following proposition due to Rheinländer $[16]$.

Proposition 2.2. [16]. Let $S$ be a locally bounded semimartingale. If $\bar{Q} \in \mathcal{M}_{e}$ has finite relative entropy and Radon-Nikodym derivative $\frac{d Q}{d P}$ is of the form (2.1) with $\int_{0}^{T} \eta_{t}^{2} d[S]_{t}$ belonging to the Orlicz space $L_{\text {exp }}^{1}(P)$ generated by Young function exp(.). Then $\int \eta d S$ is a true $Q$-martingale for all $Q \in \mathcal{M}_{e}$ with finite relative entropy, and therefore $\bar{Q}$ coincides with the MEMM.

\section{Condition B.}

There exists a positive constant $\epsilon$ such that:

$$
\exp \left[\epsilon \int_{0}^{T}\left(\frac{\mu^{2}\left(t, Y_{t}\right)}{\sigma^{2}\left(t, Y_{t}\right)}+\beta^{2}\left(t, Y_{t}\right) v^{2}\left(t, Y_{t}\right)\right) d t\right] \in L^{1}(P) .
$$

Condition $B$ will be sufficient to ensure that $Z_{T}$ is the density of the MEMM $Q_{M E}$. We now prove the following result whose idea is similar to Benth and Karlsen [3]. Here we generalize it to the time-dependent case.

Theorem 2.3. Assume

$$
\int_{0}^{T} \frac{\mu^{2}\left(t, Y_{t}\right)}{\sigma^{2}\left(t, Y_{t}\right)} d t<\infty, \quad \int_{0}^{T} \delta\left(t, Y_{t}\right) v\left(t, Y_{t}\right) d t<\infty, P-a . s .
$$

for all $t>0$ and conditions $A$ and $B$ hold. Then $Z_{T}$ in Rheinländer [16] is the density of the $M E M M Q_{M E}$.

Proof. Using that

$$
S_{t}^{-1} d S_{t}=\mu\left(t, Y_{t}\right) d t+\sigma\left(t, Y_{t}\right) d B_{t}
$$


we find:

$$
\begin{aligned}
Z_{T}= & \exp \left(-\int_{0}^{T} \frac{\mu\left(t, Y_{t}\right)}{\sigma^{2}\left(t, Y_{t}\right)} S_{t}^{-1} d S_{t}-\int_{0}^{T} \delta\left(t, Y_{t}\right) v_{y}\left(t, Y_{t}\right) d W_{t}\right. \\
& \left.+\frac{1}{2} \int_{0}^{T}\left(\frac{\mu^{2}\left(t, Y_{t}\right)}{\sigma^{2}\left(t, Y_{t}\right)}-\delta^{2}\left(t, Y_{t}\right) v_{y}^{2}\left(t, Y_{t}\right)\right) d t\right)
\end{aligned}
$$

Now let us derive an expression for the term

$$
\int_{0}^{T} \delta\left(t, Y_{t}\right) v_{y}\left(t, Y_{t}\right) d W_{t}
$$

Itô's formula holds (since we assume $v \in C^{1,2}$ )

$$
d v\left(t, Y_{t}\right)=v_{t}\left(t, Y_{t}\right) d t+\mathcal{L}_{Y} v d t+v_{y}\left(t, Y_{t}\right)\left[\beta\left(t, Y_{t}\right) d B_{t}+\delta\left(t, Y_{t}\right) d W_{t}\right]
$$

where $\mathcal{L}_{Y}$ is defined in equation (1.11).

Integrating and appealing to the $\operatorname{PDE}((1.3))$ satisfied by $v$,

$$
\begin{aligned}
v\left(T, Y_{T}\right)= & v(0, y)+\int_{0}^{T}\left[v_{t}\left(t, Y_{t}\right)+\mathcal{L}_{Y} v\left(t, Y_{t}\right)\right] d t+\int_{0}^{T} \beta\left(t, Y_{t}\right) v_{y}\left(t, Y_{t}\right) d B_{t} \\
& +\int_{0}^{T} \delta\left(t, Y_{t}\right) v_{y}\left(t, Y_{t}\right) d W_{t} \\
= & v(0, y)+\int_{0}^{T}\left[-\frac{\mu^{2}\left(t, Y_{t}\right)}{2 \sigma^{2}\left(t, Y_{t}\right)}+\frac{1}{2} \delta^{2}\left(t, Y_{t}\right) v_{y}^{2}\left(t, Y_{t}\right)\right. \\
& \left.+\frac{\mu\left(t, Y_{t}\right)\left(\beta\left(t, Y_{t}\right)\right.}{\sigma\left(t, Y_{t}\right)} v_{y}\left(t, Y_{t}\right)\right] d t+\int_{0}^{T} \beta\left(t, Y_{t}\right) v_{y}\left(t, Y_{t}\right) d B_{t}+\int_{0}^{T} \delta\left(t, Y_{t}\right) d W_{t} .
\end{aligned}
$$

Since $v(T, y)=0$ for all $y$, we obtain the relation:

$$
\begin{aligned}
\int_{0}^{T} \delta\left(t, Y_{t}\right) v_{y}\left(t, Y_{t}\right) d W_{t}= & -v(0, y)+\frac{1}{2} \int_{0}^{T} \frac{\mu^{2}\left(t, Y_{t}\right)}{\sigma^{2}\left(t, Y_{t}\right)} d t-\frac{1}{2} \int_{0}^{T} \delta^{2}\left(t, Y_{t}\right) v_{y}^{2}\left(t, Y_{t}\right) d t \\
& -\frac{\mu\left(t, Y_{t}\right)\left(\beta\left(t, Y_{t}\right)\right.}{\sigma\left(t, Y_{t}\right)} v_{y}\left(t, Y_{t}\right) d t-\int_{0}^{T} \beta\left(t, Y_{t}\right) v_{y}\left(t, Y_{t}\right) d B_{t}
\end{aligned}
$$

Inserted into the expression (2.2) for $Z_{T}$ this yields

$$
\begin{aligned}
Z_{T}= & \exp \left[-\int_{0}^{T} \frac{\mu\left(t, Y_{t}\right)}{\sigma^{2}\left(t, Y_{t}\right)} S_{t}^{-1} d S_{t}+\int_{0}^{T} \beta\left(t, Y_{t}\right) v_{y}\left(t, Y_{t}\right) d B_{t}\right. \\
& \left.+\frac{\mu\left(t, Y_{t}\right) \beta\left(t, Y_{t}\right)}{\sigma\left(t, Y_{t}\right)} v_{y}\left(t, Y_{t}\right) d t\right] .
\end{aligned}
$$

Using

$$
\sigma^{-1}\left(t, Y_{t}\right) S_{t}^{-1} d S_{t}=\mu\left(t, Y_{t}\right) \sigma^{-1}\left(t, Y_{t}\right) d t+d B_{t}
$$


gives

$$
Z_{T}=\exp \left(\int_{0}^{T}\left(\frac{\beta\left(t, Y_{t}\right)}{\sigma\left(t, Y_{t}\right)} v_{y}\left(t, Y_{t}\right)-\frac{\mu\left(t, Y_{t}\right)}{\sigma^{2}\left(t, Y_{t}\right)}\right) S_{t}^{-1} d S_{t}+v(0, y)\right)
$$

which shows that $Z_{T}$ can be written in the form (2.1) with

$$
c=v(0, y), \quad \eta_{t}=\left(\frac{\beta\left(t, Y_{t}\right)}{\sigma\left(t, Y_{t}\right)} v_{y}\left(t, Y_{t}\right)-\frac{\mu\left(t, Y_{t}\right)}{\sigma^{2}\left(t, Y_{t}\right)}\right) S_{t}^{-1} .
$$

We know already that $Z_{T}$ is the density of a martingale measure $Q$ with finite relative entropy. From Frittelli [8] it then follows that the MEMM $Q_{M E}$ exists and is moreover unique. Since

$$
d[S]_{t}=\sigma^{2}\left(t, Y_{t}\right) S_{t}^{2} d t
$$

we must have some $\epsilon>0$ that

$$
\exp \left(\epsilon \int_{0}^{T}\left[\beta\left(t, Y_{t}\right) v_{y}\left(t, Y_{t}\right)-\frac{\mu\left(t, Y_{t}\right)}{\sigma\left(t, Y_{t}\right)}\right]^{2} d t\right) \in L^{1}(P) .
$$

Condition $B$ ensures this. Consequently,

$$
\exp \left(\epsilon \int_{0}^{T} \eta_{t}^{2} d[S]_{t}\right) \in L^{1}(P)
$$

for some $\epsilon>0$. Proposition 2.2 now implies that $Z_{T}$ is the density of the MEMM $Q_{M E}$.

\section{Application to a Class of stochastic volatility models}

We consider the following class of stochastic volatility models:

$$
d Y_{t}=\alpha\left(t, Y_{t}\right) d t+\beta(t) d U_{t}, \quad \beta(t)>0,
$$

where

$$
U_{t}=\rho(t) B_{t}+\sqrt{1-\rho(t)^{2}} W_{t}
$$

and $-1<\rho(t)<1$ for a time-dependent parameter $\rho(t)$. Here $U_{t}$ is a Brownian motion correlated with $B_{t}$ (see equation (1.1)). We now introduce the following (growth) assumptions on the asset and volatility coefficients in equations (1.1) and (3.1):

$$
\left\{\begin{array}{l}
\left|\alpha\left(y^{*}\right)\right| \leq C\left|y^{*}\right|,\left|\alpha^{\prime}\left(y^{*}\right)\right| \leq C, \quad y^{*}=(t, y) \in[0, T) \times \mathbb{R} \\
\left|\frac{\mu\left(y^{*}\right)}{\sigma\left(y^{*}\right)}\right| \leq C\left|\left(y^{*}\right)\right|,\left|\left(\frac{\mu\left(y^{*}\right)}{\sigma\left(y^{*}\right)}\right)^{\prime}\right| \leq C, \quad y^{*}=(t, y) \in[0, T) \times \mathbb{R} .
\end{array}\right.
$$

We prove that the conditions in (3.2) are sufficient for the existence of a unique quadratically growing classical solution $v=v(t, y)$, with a linearly growing derivative $v_{y}(t, y)$, of the semilinear PDE:

$$
-v_{t}-\frac{1}{2} \beta^{2}(t) v_{y y}+F\left(t, y, v_{y}\right)=0, \quad(t, y) \in[0, T) \times \mathbb{R},
$$


with terminal condition

$$
v(T, y)=0, \quad y \in \mathbb{R} .
$$

The non-linear function $F:[0, T) \times \mathbb{R} \times \mathbb{R} \rightarrow \mathbb{R}$ is defined by:

$$
F(t, y, p)=\frac{1}{2} \delta^{2}(t) p^{2}-\left(\alpha(t, y)-\frac{\mu(t, y) \beta(t) \rho(t)}{\sigma(t, y)}\right) p-\frac{\mu^{2}(t, y)}{\sigma^{2}(t, y)},
$$

and $\delta^{2}(t)=\beta^{2}(t)\left(1-\rho^{2}(t)>0\right.$ is a time-dependent parameter. Note that the problem (3.3)-(3.4) corresponds to (1.3)-(1.4) with $\delta(t, y)=\beta(t) \sqrt{1-\rho^{2}(t)}$ and $\beta(t, y)=\beta(t) \rho(t)$ for all $(t, y)$.

We will prove that the linear growth of the derivative $v_{y}$ implies that Conditions $A$ and $B$ hold. Hence, under the stated conditions stated in (3.2) on the asset price model (1.1) and the volatility model (3.1), we have the existence of the MEMM $Q_{M E}$ with density as in equation (1.6) or equivalently (2.3). The Stein-Stein volatility model is covered by the theory of this section, and for this model an explicit solution of (3.3)-(3.4) can be found.

3.1. Well-posedness of the semilinear PDE. Since $p \longrightarrow F(t, y, p)$ is not globally Lipschitz continuous, the existence of a classical solution to equations (3.3) and (3.4) cannot be found directly in the literature. Here we will use the approach taken by Benth and Karlsen [3] by considering a certain sequence of approximating PDEs which are the HJB-equations of certain stochastic control problems for which the existence of smooth solutions is well known.

Introduce the function

$$
L:[0, T) \times \mathbb{R} \times \mathbb{R} \longrightarrow \mathbb{R}
$$

by:

$$
L(t, y, q)=\max _{p \in \mathbb{R}}\{-q p-F(t, y, p)\} .
$$

Next we can easily check that

$$
\begin{aligned}
L(t, y, q) & \\
& =\max _{p \in \mathbb{R}}\{-q p-F(t, y, p)\} . \\
& =\max _{p \in \mathbb{R}}\left\{-q p-\left[\frac{1}{2} \delta^{2}(t, y) p^{2}-\left(\alpha(t, y)-\frac{\mu(t, y) \beta(t) \rho(t)}{\sigma(t, y)}\right) p-\frac{\mu^{2}(t, y)}{\sigma^{2}(t, y)}\right]\right\} .
\end{aligned}
$$

We do the following: completing the square in $p$ and noting that the maximum expression of $p$ is given by:

$$
p=\frac{1}{\delta^{4}(t, y)}\left[\left(\alpha(t, y)-\frac{\mu(t, y) \beta(t) \rho(t)}{\delta(t, y)}\right)\right] .
$$


We then simplify the above expression to obtain

$$
L(t, y, q)=\frac{1}{2 \delta^{2}(t, y)}\left(q-\left[\alpha(t, y)-\frac{\mu(t, y) \beta(t) \rho(t)}{\delta(t, y)}\right]\right)^{2}+\frac{1}{2} \frac{\mu^{2}(t, y)}{\sigma^{2}(t, y)} .
$$

The following duality relation can be checked using the above technique:

$$
F(t, y, p)=\max _{q \in \mathbb{R}}\{-q p-L(t, y, p)\} .
$$

Consider the auxiliary function

$$
F^{k}:[0, T) \times \mathbb{R} \times \mathbb{R} \longrightarrow \mathbb{R}
$$

defined for each natural number $k$ by:

$$
F^{k}(t, y, p)=\max _{|q| \leq k}\{-q p-L(t, y, p)\} .
$$

From

$$
\left|\alpha\left(y^{*}\right)-\frac{\mu\left(y^{*}\right) \beta(t) \rho(t)}{\sigma\left(y^{*}\right)}\right| \leq C\left|y^{*}\right|, \quad y^{*}=(t, y) \in[0, T) \times \mathbb{R},
$$

we find

$$
|L(t, y, p)| \leq C\left(q^{2}+y^{2}\right), \quad(t, y, q) \in[0, T) \times \mathbb{R} \times \mathbb{R} .
$$

Moreover, since

$$
\begin{aligned}
L_{y^{*}}\left(y^{*}, q\right)= & \frac{-1}{\delta^{2}(t, y)}\left(q-\left[\alpha\left(y^{*}\right)-\frac{\mu\left(y^{*}\right) \beta(t) \rho(t)}{\delta\left(y^{*}\right)}\right]\right) \\
& \times\left[\alpha^{\prime}\left(y^{*}\right)-\left(\frac{\mu\left(y^{*}\right)}{\sigma\left(y^{*}\right)}\right)^{\prime} \beta(t) \rho(t)\right]+\frac{1}{2}\left(\frac{\mu\left(y^{*}\right)}{\sigma\left(y^{*}\right)}\right)^{\prime},
\end{aligned}
$$

it follows from (3.2) that

$$
\left|L_{y^{*}}\left(y^{*}, q\right)\right| \leq C\left(|q|+\left|y^{*}\right|\right), \quad\left(y^{*}, q\right) \in[0, T) \times \mathbb{R} \times \mathbb{R},
$$

where $y^{*}=(t, y)$.

In particular, $|L|$ and $\left|L_{y^{*}}\right|$ are of polynomial growth in $y$ uniformly in $q$ when $|q| \leq k$. Hence, by classical theory (Fleming and Soner [7], Theorem 4.3 on p.169) there exists a unique polynomially growing solution

$$
v^{k} \in C^{1,2}([0, T) \times \mathbb{R} \cap C([0, T] \times \mathbb{R}),
$$

of the semilinear PDE

$$
-v_{t}-\frac{1}{2} \beta^{2}(t) v_{y y}^{k}+F^{k}\left(t, y, v_{y}^{k}\right)=0, \quad(t, y) \in[0, T) \times \mathbb{R},
$$


with terminal condition $v^{k}(T, y)=0$, for all $y \in \mathbb{R}$.

We need to derive estimates on $v_{t}^{k}$ and $v_{y}^{k}$ that are independent of $k$. For simplicity, in some cases we shall consider $\beta(t)=\beta$ as a constant.

Lemma 3.1. There exists a constant $C$ that is independent of $k$ such that

$$
\left|v_{y}^{k}(t, y)\right| \leq C(1+|y|), \quad(t, y) \in[0, T) \times \mathbb{R} .
$$

Proof. This follows as in (Benth and Karlsen [3], Lemma (3.1)). However, we need to clarify some time-dependent parameters in the proof. From standard theory Fleming and Soner [7], $v^{k}$ can be represented as the solution of the stochastic control problem:

$$
v^{k}(t, y)=\inf _{q \in \mathcal{A}_{t}^{k}} \mathbb{E}\left[\int_{t}^{T} L\left(s, \widehat{Y}_{s}, q_{s}\right) d s \mid \widehat{Y}_{t}=y\right]
$$

where $\mathcal{A}_{t}^{k}$ denotes the set of adapted control processes that are bounded by $k$, and

$$
d \widehat{Y}_{t}=q_{t} d t+\beta(t) d U_{t} .
$$

Moreover, an optimal control for (3.7) is Markov and is given in feedback form by:

$$
q_{k}^{*}(t, y)=\arg \min _{|q| \leq k}\left\{q v_{y}(t, y)+L(t, y, q)\right\} .
$$

As a result we obtain:

$$
v^{k}(t, y)=\mathbb{E}\left[\int_{t}^{T} L\left(s, \widehat{Y}_{s}^{*}, q_{k}^{*}\left(s, \widehat{Y}_{s}^{*}\right)\right) d s \mid \widehat{Y}_{t}^{*}=y\right],
$$

where $\widehat{Y}_{t}^{*}$ solves (3.8) with the control $q_{t}=q_{k}^{*}\left(s, \widehat{Y}_{s}^{*}\right)$. From standard theory (Fleming and Soner [7], Lemma 11.4 on page 209), we have:

$$
v_{y}^{k}(t, y)=\mathbb{E}\left[\int_{t}^{T} L_{y}\left(s, \widehat{Y}_{s}^{*}, q_{k}^{*}\left(s, \widehat{Y}_{s}^{*}\right)\right) d s \mid \widehat{Y}_{t}^{*}=y\right]
$$

Using the definition of $L(t, y, q)$ and its derivative $L_{y}(t, y, q)$ we have that

$$
\left|L_{y}(t, y, q)\right| \leq C \sqrt{L(t, y, q)}
$$

for some constant $C$. Thus, by the Cauchy-Schwartz inequality and the fact that $L(t, y, 0) \leq$ $C y^{2}$ for a constant $C$ independent of $k$,

$$
\begin{aligned}
v_{y}^{k}(t, y) & \leq \mathbb{E}\left[\int_{t}^{T}\left|L_{y}\left(s, \widehat{Y}_{s}^{*}, q_{k}^{*}\left(s, \widehat{Y}_{s}^{*}\right)\right)\right| d s \mid \widehat{Y}_{t}^{*}=y\right] \\
& \leq(T-t)^{\frac{1}{2}} \mathbb{E}\left[\int_{t}^{T}\left|L_{y}\left(s, \widehat{Y}_{s}^{*}, q_{k}^{*}\left(s, \widehat{Y}_{s}^{*}\right)\right)\right|^{2} d s \mid \widehat{Y}_{t}^{*}=y\right]^{\frac{1}{2}}, \\
& \leq C \mathbb{E}\left[\int_{t}^{T} L\left(s, \widehat{Y}_{s}^{*}, q_{k}^{*}\left(s, \widehat{Y}_{s}^{*}\right)\right) d s \mid \widehat{Y}_{t}^{*}=y\right]^{\frac{1}{2}},
\end{aligned}
$$




$$
\begin{aligned}
& \leq C \mathbb{E}\left[\int_{t}^{T} L\left(s, \widehat{Y}_{s}, 0\right) d s \mid \widehat{Y}_{t}=y\right]^{\frac{1}{2}}, \\
& \leq C \mathbb{E}\left[\int_{t}^{T} \widehat{Y}_{s}^{2} d s \mid \widehat{Y}_{t}=y\right]^{\frac{1}{2}}, \\
& \leq C(1+|y|),
\end{aligned}
$$

where we have used the fact that for the control $q=0, \widehat{Y}_{s}=y+\beta\left(U_{s}-U_{t}\right)$, for $s \geq t$. The constant $C$ changes from line to line in the above estimation process, but is always independent of $k$.

Lemma 3.2. There exists a constant $C$ that is independent of $k$ such that

$$
\left|v_{t}^{k}(t, y)\right| \leq C\left(1+|y|^{2}\right), \quad(t, y) \in[0, T) \times \mathbb{R} .
$$

Proof. The proof is essentially the same as for (Benth and Karlsen [3], Lemma (3.2)). We only have to clarify a few points concerning the time-dependence of the some parameters. Let

$$
\widehat{q}_{k}(t, y):=\arg \min _{q \in \mathbb{R}}\left\{q v_{y}^{k}+L(t, y, q)\right\} .
$$

We can easily check that

$$
\widehat{q}_{k}(t, y)=\tilde{\alpha}(t, y)-\delta^{2}(t) v^{k}(t, y)
$$

where

$$
\tilde{\alpha}(t, y)=\alpha(t, y)-\frac{\mu(t, y) \beta \rho(t)}{\sigma(t, y)} .
$$

From the growth conditions on $\alpha(t, y), \frac{\mu(t, y)}{\sigma(t, y)}$ and the estimate on $v_{y}^{k}(t, y)$ in Lemma 3.1 , there exists a constant $C$ independent of $k$ such that

$$
\left|\widehat{q}_{k}(t, y)\right| \leq C(1+|y|) .
$$

Note that

$$
q_{k}^{*}(t, y)=k \vee \widehat{q}_{k}(t, y) \wedge(-k),
$$

where $q^{*}$ is defined in equation (3.9). Therefore

$$
\left|q_{k}^{*}(t, y)\right| \leq C(1+|y|),
$$

where $C$ appears in equation (3.11). It is important for us later to have a bound for $q^{*}$ which is independent of $k$. A change of time in equation (3.10) yields:

$$
v^{k}(t, y)=\mathbb{E}\left[\int_{0}^{T-t} L\left(t+s, \widehat{Y}_{t+s}^{*}, q_{k}^{*}\left(t+s, \widehat{Y}_{t+s}^{*}\right)\right) d s \mid \widehat{Y}_{t}^{*}=y\right] .
$$


If $\bar{U}_{s}:=U_{t+s}$, then $\bar{U}$ becomes a Brownian motion since it is a time change of $U$. Assume that $\bar{q}_{t}$ belongs to the set of adapted stochastic processes bounded on $[0, T-t]$ by $k$, which are now denoted by $\overline{\mathcal{A}}^{k}$. Consider the optimal control problem with criterion function:

$$
\mathbb{E}\left[\int_{0}^{T-t} L\left(s, \bar{Y}_{s}, \bar{q}_{s}\right) d s \mid \bar{Y}_{0}=y\right]
$$

and dynamics $d \bar{Y}_{s}=\bar{q}_{s} d s+\beta d \bar{U}_{s}$.

From standard theory Fleming and Soner [7], there exists an optimal feedback control $\bar{q}_{k}^{*}$ solving this problem, and it holds that $\bar{Y}_{s}^{*}=\widehat{Y}_{s}^{*}$ in distribution since $\bar{q}_{k}^{*}=q_{k}^{*}$. Thus

$$
v^{k}(t, y)=\mathbb{E}\left[\int_{0}^{T-t} L\left(s, \bar{Y}_{s}^{*}, \bar{q}^{*}\left(s, \bar{Y}_{s}^{*}\right)\right) d s \mid \bar{Y}_{0}=y\right]
$$

Following the estimation procedure in Fleming and Soner [7] and using equation (3.5) we get:

$$
\begin{aligned}
\left|v_{t}^{k}(t, y)\right| & \leq C \mathbb{E}\left[\left(\bar{q}_{k}^{*}\left(T-t, \bar{Y}_{T-t}\right)\right)^{2}+\bar{Y}_{T-t}^{2} \mid \bar{Y}_{0}=y\right] \\
& \leq C\left(1+\mathbb{E}\left[\bar{Y}_{T-t}^{2} \mid \bar{Y}_{0}=y\right]\right)
\end{aligned}
$$

where (3.12) was used to derive the second inequality.

Estimating $\mathbb{E}\left[\bar{Y}_{t}^{2}\right]$ for a $t \in[0, T]$. Using Itô's formula and (3.12), we find for the stopping times $s_{n}=\tau_{n} \wedge t$, where

$$
\begin{gathered}
\tau_{n}=\inf \left\{t \geq 0 \text { and }\left|\bar{Y}_{t}\right| \geq n\right\} \\
\mathbb{E}\left[\bar{Y}_{s_{n}}^{2}\right] \leq y^{2}+\int_{0}^{t}\left(\beta^{2}+2 C\right) d s+2 C \int_{0}^{t} \mathbb{E}\left[\bar{Y}_{s}^{2}\right] d s .
\end{gathered}
$$

Since $s_{n} \uparrow t$ when $n \rightarrow \infty$, we get from Fatou's lemma and Gronwall's inequality that

$$
\mathbb{E}\left[\bar{Y}_{t}^{2}\right] \leq C\left(1+|y|^{2}\right) .
$$

The constant $C$ may have changed from line to line in the above estimation process, but is always independent of $k$. The lemma now follows from equations (3.13) and (3.15).

The following theorem gives a result about the existence and uniqueness of the classical solution $v$. A sketch of the existence proof is given in Benth and Karlsen [3] and the uniqueness assertion of the theorem follows from [5]. As in Benth and Karlsen [3] it is noted that to identify $Z_{T}$ in equation (1.6) as the density of the $M E M M Q_{M E}$ we only need to show that there exists at least one solution, hence uniqueness is strictly speaking not needed in the identification process.

Theorem 3.3. Suppose the conditions in (3.2) hold. Then there exists a unique classical solution:

$$
v \in C^{1,2}([0, T) \times \mathbb{R}) \cap C([0, T] \times \mathbb{R})
$$

of the terminal value problem (3.3)-(3.4). Moreover, $v(t, y)$ is at most quadratically growing in $y$ while the derivative $v_{y}(t, y)$ is at most linearly growing in $y$. 
Proof. The proof follows as in (Benth and Karlsen [3], Theorem 3.3).

3.2. Verification of Conditions $A$ and $B$. Now we prove that Conditions $A$ and $B$ hold under the assumptions stated in (3.2) for the asset and volatility dynamics (1.1)-(3.1).

Proposition 3.4. Assume that the conditions stated in (3.2) hold and $\beta(t)=\beta$ a constant. Then Condition A holds for the model (1.1)-(3.1).

Proof. We make some modifications in the proof in (Benth and Karlsen [3], Proposition (3.4)). Following condition (1) in theorem (2.1), we need to prove that

$$
\sup _{n} \mathbb{E}_{Q_{n}}\left[\int_{0}^{T_{n}} Y_{s}^{2} d s\right]<\infty
$$

where $Q_{n}$ and $T_{n}$ are defined just before (2.1). Define the process

$$
\tilde{U}_{t}^{n}=\rho(t) \tilde{B}_{t \Lambda T_{n}}+\sqrt{1-\rho(t)} \tilde{W}_{t \Lambda T_{n}} .
$$

Note that $\tilde{U}^{n}$ is a Brownian motion under $Q_{n}$. For $t \leq T_{n}$, it holds

$$
d Y_{t}=\tilde{\alpha}\left(t, Y_{t}\right) d t+\beta d \tilde{U}_{t}^{n},
$$

with

$$
\tilde{\alpha}(t, y)=\alpha(t, y)-\frac{\mu(t, y) \beta \rho(t)}{\sigma(t)}-\beta^{2}(t)\left(1-\rho(t)^{2}\right) v_{y}^{2}(t, y) .
$$

From the assumptions (3.2) and the linear growth of $v_{y}$ we find

$$
|\tilde{\alpha}(t, y)| \leq C(1+|y|),
$$

for some positive constant $C$. Itô's formula yields for $t \leq T_{n}$,

$$
Y_{t}^{2}=y^{2}+\beta^{2} t+2 \int_{0}^{t} Y_{s} \tilde{\alpha}\left(s, Y_{s}\right) d s+2 \int_{0}^{t} Y_{s} d \tilde{U}_{s}^{n},
$$

and we get

$$
\int_{0}^{T_{n}} Y_{t}^{2} d t=y^{2} T_{n}+\frac{1}{2} \beta^{2} T_{n}^{2}+2 \int_{0}^{T_{n}} \int_{0}^{t} Y_{s} \tilde{\alpha}\left(s, Y_{s}\right) d s d t+2 \int_{0}^{T_{n}} \int_{0}^{t} Y_{s} d \tilde{U}_{s}^{n} d t .
$$

Taking the expectation with respect to $Q_{n}$ and using $T_{n} \leq T$,

$$
\begin{aligned}
\mathbb{E}_{Q_{n}}\left[\int_{0}^{T_{n}} Y_{t}^{2} d s\right] \leq & y^{2} T+\frac{1}{2} \beta^{2} T^{2}+2 \mathbb{E}_{Q_{n}}\left[\int_{0}^{T_{n}} \int_{0}^{t} Y_{s} \tilde{\alpha}\left(s, Y_{s}\right) d s d t\right] \\
& +2 \mathbb{E}_{Q_{n}}\left[\int_{0}^{T_{n}} \int_{0}^{t} Y_{s} d \tilde{U}_{s}^{n} d t\right] .
\end{aligned}
$$

Appealing to the Cauchy-Schwartz inequality we can estimate the last term on the righthand side as follows:

$$
\mathbb{E}_{Q_{n}}\left[\int_{0}^{T_{n}} \int_{0}^{t} Y_{s} d \tilde{U}_{s}^{n} d t\right]=\mathbb{E}_{Q_{n}}\left[\int_{0}^{\infty} 1_{t \leq T_{n}} \int_{0}^{t} Y_{s} d \tilde{U}_{s}^{n} d t\right]
$$




$$
\begin{aligned}
& \leq \mathbb{E}_{Q_{n}}\left[\int_{0}^{\infty} 1_{t \leq T_{n}}\right]^{\frac{1}{2}} \mathbb{E}_{Q_{n}}\left[\int_{0}^{\infty} 1_{t \leq T_{n}}\left(\int_{0}^{t} Y_{s} d \tilde{U}_{s}^{n}\right)^{2} d t\right]^{\frac{1}{2}} \\
& \leq \sqrt{T} \mathbb{E}_{Q_{n}}\left[\int_{0}^{T_{n}}\left(\int_{0}^{t} Y_{s} d \tilde{U}_{s}^{n}\right)^{2} d t\right]^{\frac{1}{2}} .
\end{aligned}
$$

From the Itô isometry and the algebraic inequality $2 a b \leq a^{2}+b^{2}$, there exists a positive constant $C$ such that:

$$
\begin{aligned}
\mathbb{E}_{Q_{n}}\left[\int_{0}^{T_{n}} \int_{0}^{t} Y_{s} d \tilde{U}_{s}^{n} d t\right] & \leq \frac{1}{\sqrt{T}}\left(1+\left(\int_{0}^{T} \mathbb{E}_{Q_{n}}\left[\int_{0}^{t} Y_{s}^{2} d s\right] d t\right)^{2}\right)^{\frac{1}{2}} \\
& \leq C\left(1+\left(\int_{0}^{T} \mathbb{E}_{Q_{n}}\left[\int_{0}^{t} Y_{s}^{2} d s\right] d t\right) .\right.
\end{aligned}
$$

Since $T_{n} \uparrow T$ a.s. when $n \rightarrow \infty$, it follows from Fatou's lemma together with the linear growth (3.16) of the coefficient $\tilde{\alpha}$ that

$$
\begin{aligned}
\mathbb{E}_{Q_{n}}\left[\int_{0}^{T} Y_{s}^{2} d s\right] \leq & \lim _{n \rightarrow \infty} \operatorname{E}_{Q_{n}}\left[\int_{0}^{T_{n}} Y_{s}^{2} d s\right] \\
\leq & y^{2} T+\frac{1}{2} \beta^{2} T^{2}+2 C \lim \inf _{n \rightarrow \infty} \mathbb{E}_{Q_{n}}\left[\int_{0}^{T_{n}} \int_{0}^{t}\left|Y_{s}\right|\left(1+\left|Y_{s}\right|\right) d s d t\right] \\
& +C\left(1+\int_{0}^{T} \mathbb{E}_{Q_{n}} \int_{0}^{t} Y_{s}^{2} d s d t\right) \\
\leq & C \lim _{n \rightarrow \infty}\left(1+\int_{0}^{T} \mathbb{E}_{Q_{n}} \int_{0}^{t} Y_{s}^{2} d s d t\right) \\
\leq & C\left(1+\int_{0}^{T} \sup _{n} \mathbb{E}_{Q_{n}} \int_{0}^{t} Y_{s}^{2} d s d t\right)
\end{aligned}
$$

where the constant $C$ has possibly changed from line to line in the estimation process. Hence

$$
\sup _{n} \mathbb{E}_{Q_{n}}\left[\int_{0}^{T} Y_{s}^{2} d s\right] \leq C\left(1+\int_{0}^{T} \sup _{n} \mathbb{E}_{Q_{n}} \int_{0}^{t} Y_{s}^{2} d s d t\right)
$$

and from Gronwall's inequality it follows that

$$
\sup _{n} \mathbb{E}_{Q_{n}}\left[\int_{0}^{T} Y_{s}^{2} d s\right] \leq C\left(1+e^{C T}\right) .
$$

But then we have

$$
\sup _{n} \mathbb{E}_{Q_{n}}\left[\int_{0}^{T_{n}} Y_{s}^{2} d s\right] \leq \sup _{n} \mathbb{E}_{Q_{n}}\left[\int_{0}^{T} Y_{s}^{2} d s\right] \leq C\left(1+e^{C T}\right),
$$

and the proposition is proved.

Before we prove that Condition $B$ holds, let us state the following lemma with slight changes in parameters, which yields a bound on the moments of $Y_{t}$ starting from zero. 
Lemma 3.5. Let $Y_{0}=0$. Suppose

$$
\left|\alpha\left(y^{*}\right)\right| \leq C\left|y^{*}\right|
$$

for all

$$
y^{*}=(t, y) \in[0, T) \times \mathbb{R},
$$

then

$$
\mathbb{E}\left[Y_{t}^{2 n}\right] \leq 2^{-2 n} \frac{(2 n) !}{n !}\left(\frac{\beta^{2}}{C}\right)^{n}\left(e^{2 C t}-1\right) .
$$

Proof. The proof follows as in (Benth and Karlsen [3], Lemma (3.5)).

Proposition 3.6. Assume that the conditions stated in (3.2) hold. Then Condition B holds for the model (1.1)-(3.1).

Proof. To prove Condition $B$, first observe that, from the assumptions on

$$
\mu(t, y), \quad \sigma(t, y), \quad \delta(t, y)
$$

in (3.2) and the linear growth on $v_{y}$, we have

$$
\frac{\mu^{2}\left(t, Y_{t}\right)}{\sigma^{2}\left(t, Y_{t}\right)}+\delta^{2}\left(t, Y_{t}\right) v_{y}^{2}\left(t, Y_{t}\right) \leq C\left(1+Y_{t}^{2}\right)
$$

Before proceeding further, let us show that

$$
\left|Y_{t}(y)\right| \leq\left|Y_{t}(0)\right|+|y| e^{C t}
$$

where $Y_{t}(y)$ is the process $Y_{t}$ starting in $y$ at time zero, and $C$ is the growth rate $\alpha$, as in (Benth and Karlsen [3], Proposition (3.6)). From Ikeda and Watanabe [15] we find that (since $\alpha$ is assumed to be differentiable)

$$
\frac{\partial}{\partial y} Y_{t}(y)=1+\int_{0}^{t} \alpha^{\prime}\left(s, Y_{s}(y)\right) \frac{\partial}{\partial y} Y_{s}(y) d s
$$

and thus

$$
\frac{\partial}{\partial y} Y_{t}(y)=\exp \left(\int_{0}^{t} \alpha^{\prime}\left(s, Y_{s}(y)\right) d s\right)
$$

Since $\left|\alpha^{\prime}(t, y)\right| \leq C$, the desired bound follows by using the fundamental theorem of calculus. It is therefore sufficient to prove that:

$$
\exp \left(\epsilon \int_{0}^{T} Y_{s}^{2} d s\right) \in L^{1}(P)
$$

for $\epsilon>0$ when $Y$ is starting at zero. Using Hölder's inequality (with $p=\frac{n}{n-1}$ and $q=\frac{1}{n}$ ) and Lemma 3.5 we find:

$$
\mathbb{E}\left[\exp \left(\epsilon \int_{0}^{T} Y_{s}^{2}(0) d s\right)\right]=1+\sum_{n=1}^{\infty} \frac{\epsilon^{n}}{n !} \mathbb{E}\left[\left(\int_{0}^{T} Y_{s}^{2}(0) d s\right)^{n}\right]
$$




$$
\begin{aligned}
& \leq 1+T^{-1} \sum_{n=1}^{\infty} \frac{(\epsilon T)^{n}}{n !} \mathbb{E}\left[\int_{0}^{T} Y_{s}^{2 n} d s\right] \\
& \leq 1+\sum_{n=1}^{\infty}(\epsilon k(t))^{n} \frac{(2 n) !}{(n !)^{2}}
\end{aligned}
$$

for some time-dependent parameter $k(t)$ depending on $\beta(t), C$, and $T$. By choosing $\epsilon$ sufficiently small (that is, $\epsilon<\frac{1}{4 k(t)}$ ), this series becomes convergent. Hence, Condition $B$ holds.

3.3. The Stein-Stein volatility model. We now apply our general results to a version of the time-dependent Stein-Stein stochastic volatility model [3]. Assume $\frac{\mu\left(y^{*}\right)}{\sigma\left(y^{*}\right)}=\xi(t) y^{*}$ for some time-dependent parameter different from zero and $y^{*}=(t, y)$. Let the volatility $Y_{t}$ follow an Ornstein-Uhlenbeck-type process:

$$
d Y_{t}=\left(m-\alpha(t) Y_{t}\right) d t+\beta(t) \rho(t) d B(t)+\beta(t) \sqrt{1-\rho^{2}(t)} d W_{t},
$$

where $m$ is a positive constant and $\alpha(t), \beta(t)$ are positive time-dependent parameters with $-1<\rho(t)<1$. The parameters of this model satisfy the conditions in (3.2), and thus we have that the density of the MEMM $Q_{M E}$ for the Stein-Stein model is given by:

$$
Z_{T}=\exp \left(\int_{0}^{T}\left(\frac{\beta(t) \rho(t)}{\sigma\left(t, Y_{t}\right)} v_{y}\left(t, Y_{t}\right)-\frac{\xi(t) Y_{t}}{\sigma\left(t, Y_{t}\right)}\right) S_{t}^{-1} d S_{t}+v(0, y)\right)
$$

where $v$ is the solution to the semilinear PDE (1.3), which in the present context reads:

$$
\begin{aligned}
& -v_{t}-\frac{1}{2} \beta^{2}(t) v_{y y}+\frac{1}{2} \beta^{2}(t)\left(1-\rho^{2}(t)\right)\left(v_{y}\right)^{2} \\
& -(m-(\alpha(t)+\xi(t) \beta(t) \rho(t)) y) v_{y}-\frac{1}{2} \xi^{2}(t) y^{2}=0 .
\end{aligned}
$$

A solution to equation (3.17) is derived in the next lemma.

Lemma 3.7. The solution $v$ to (3.17), satisfying the terminal condition $v(T, y)=0$ for all $y$, is given by

$$
v(t, y)=a(t) y^{2}+b(t) y+c(t)
$$

where

$$
a(t)=a_{1}(t) \tanh \left(\int_{t}^{T} a_{2}(s) d s+a_{3}(t)\right)+a_{4}(t)
$$




$$
\begin{aligned}
b(t)= & -2 m \int_{t}^{T} a(s) \exp \int_{t}^{s}(-(\alpha(u)+\xi(u) \beta(u) \rho(u)) \\
& \left.+2 \beta^{2}(u)\left(1-\rho^{2}(u)\right) a(u) d u\right) d s, \\
c(t)= & \int_{t}^{T} \beta^{2}(s) a(s) d s+m \int_{t}^{T} b(s) d s \\
& -\frac{1}{2} \int_{t}^{T} \beta^{2}(s)\left(1-\rho^{2}(s)\right) b^{2}(s) d s,
\end{aligned}
$$

and

$$
\begin{gathered}
a_{1}(t)=\frac{\sqrt{\xi^{2}(t) \beta^{2}(t)+2 \xi(t) \alpha(t) \beta(t) \rho(t)+\alpha^{2}(t)}}{2 \beta^{2}(t)\left(1-\rho^{2}(t)\right)}, \\
a_{2}(t)=\sqrt{\xi^{2}(t) \beta^{2}(t)+2 \xi(t) \alpha(t) \beta(t) \rho(t)+\alpha^{2}(t)} \\
a_{3}(t)=\frac{1}{2} \ln \left(\frac{a_{2}(t)+\alpha(t)+\xi(t) \beta(t) \rho(t)}{a_{2}(t)-(\alpha(t)+\xi(t) \beta(t) \rho(t))}\right), \quad a_{4}(t)=-\frac{\alpha(t)+\xi(t) \beta(t) \rho(t)}{2 \beta^{2}(t)\left(1-\rho^{2}(t)\right)} .
\end{gathered}
$$

Proof. Substituting the expressions derived from equation (3.18) into the PDE (3.17), we derive the following differential equations for the coefficients $a(t), b(t)$ and $c(t)$ :

$$
\begin{aligned}
a^{\prime}(t) & =-\frac{1}{2} \xi^{2}(t)+2(\alpha(t)+\xi(t) \beta(t) \rho(t)) a(t)+2 \beta^{2}(t)\left(1-\rho^{2}(t)\right) a^{2}(t), \\
b^{\prime}(t) & =\left((\alpha(t)+\xi(t) \beta(t) \rho(t))-2 \beta^{2}(t)\left(1-\rho^{2}(t)\right) a(t)\right) b(t)-2 m a(t), \\
c^{\prime}(t) & =-\beta^{2}(t) a(t)-m b(t)+\frac{1}{2} \beta^{2}(t)\left(1-\rho^{2}(t)\right) b^{2}(t) .
\end{aligned}
$$

The terminal conditions are $a(T)=b(T)=c(T)=0$, since $v(T, y)=0$ for all $y \in \mathbb{R}$. It can be easily verified that $a(t), b(t)$ and $c(t)$ given in the Lemma are solutions, and hence the proof is complete.

Now it is clear that $a_{3}(t)$ is well defined for all possible choices of the parameters. Observe that:

$$
\frac{a_{2}(t)+\alpha(t)+\xi(t) \beta(t) \rho(t)}{a_{2}(t)-(\alpha(t)+\xi(t) \beta(t) \rho(t))}
$$

is positive since $|\rho(t)|<1$ and therefore

$$
a_{2}(t)>|\alpha(t)+\xi(t) \beta(t) \rho(t)| .
$$

Since $v_{y}(t, y)=2 a(t) y+b(t)$, we find that the density of the MEMM $Q_{M E}$ takes the form

$$
Z_{T}=\exp \left(v(0, y)+\int_{0}^{T}\left(\frac{(2 \beta(t) \rho(t) a(t)-\xi(t)) Y_{t}}{\sigma\left(t, Y_{t}\right)}+\frac{\beta(t) \rho(t) b(t)}{\sigma\left(t, Y_{t}\right)}\right) S_{t}^{-1} d S_{t}\right) .
$$

An explicit density for the Stein-Stein model was derived and analysed in Benth and Karlsen [3] and Rheinländer [16] with $\mu(y)=\mu y^{2}$ and $\sigma(y)=\sigma y$, for $\mu, \sigma$ constants. 


\section{Application to the Heston volatility model}

Another popular volatility model is provided by Heston [9] and used in Benth and Karlsen [3] which is generalized here by assuming that the volatility $Y_{t}$ follows the process

$$
d Y_{t}=\left(\lambda(t) Y_{t}^{-1}-\alpha(t) Y_{t}\right) d t+\beta(t) \rho(t) d B_{t}+\beta(t) \sqrt{1-\rho(t)} d W_{t},
$$

where $\lambda(t), \alpha(t)$ and $\beta(t)$ are positive time-dependent parameters and $-1<\rho(t)<1$. If we let $X_{t}:=Y_{t}^{2}$, we see from Itó's formula that $X_{t}$ is a Bessel process. Note that in this volatility model, we have complete information since $\lambda$ and $\alpha$ are assumed to be positive (which implies that the process $Y$ is positive). We suppose that the relation between the expected return and variance is $\frac{\mu\left(y^{*}\right)}{\sigma\left(y^{*}\right)}=\xi(t) y^{*}$ for $y^{*}=(t, y)$ and $\xi(t) \neq 0$. Unfortunately, the volatility dynamics $Y_{t}$ does not satisfy the assumptions in (3.2). However, as we will demonstrate, one can still derive a solution to the associated semilinear PDE (1.3) :

$$
\begin{aligned}
-v_{t} & -\frac{1}{2} \beta^{2}(t) v_{y y}+\frac{1}{2} \beta^{2}(t)\left(1-\rho^{2}(t)\right)\left(v_{y}\right)^{2}-\left(\lambda(t) y^{-1}\right. \\
- & (\alpha(t)+\xi(t) \beta(t) \rho(t)) y) v_{y}-\frac{1}{2} \xi^{2}(t) y^{2}=0 .
\end{aligned}
$$

The following lemma provides us with the solution to the PDE (4.2).

Lemma 4.1. The solution $v$ to equation (4.2), satisfying the terminal condition $v(T, y)=0$ for all $y$, is given by:

$$
v(t, y)=a(t) y^{2}+b(t)
$$

where $a(t)$ is given in Lemma 3.7 and

$$
b(t)=\int_{t}^{T}\left(2 \lambda(s)+\beta^{2}(s)\right) a(s) d s .
$$

Proof. Inserting the expression (4.3) into equation (4.2) leads to

$$
\begin{aligned}
a^{\prime}(t)= & -\frac{1}{2} \xi^{2}(t)+2(\alpha(t)+\xi(t) \beta(t) \rho(t)) a(t) \\
& +2 \beta^{2}(t)\left(1-\rho^{2}(t)\right) a^{2}(t), \\
b^{\prime}(t)= & -\left(2 \lambda(t)+\beta^{2}(t)\right) a(t),
\end{aligned}
$$

with terminal conditions $a(T)=b(T)=0$. Note that $a(t)$ solves the same differential equation as for Stein-Stein model, (see the proof of Lemma 3.7 in this paper). The lemma now follows.

The candidate density for the MEMM $Q_{M E}$ becomes:

$$
Z_{T}=\exp \left(v(0, y)+\int_{0}^{T}\left(\frac{(2 \beta(t) \rho(t) a(t)-\xi(t)) Y_{t}}{\sigma\left(t, Y_{t}\right)}\right) S_{t}^{-1} d S_{t}\right) .
$$

Note the similarity with the MEMM for Stein-Stein model when $m=0$. The arguments needed to verify that equation (4.4) is the density for the MEMM in the Heston model, 
however slightly different. We verify Conditions $A$ and $B$ under an extra assumption on the parameters in the Heston model.

Proposition 4.2. Assume $\alpha(t)>\beta(t) \xi(t)$ and that functions $\lambda(t)$ and $\beta(t)$ are bounded. Then Conditions $A$ and $B$ hold for the Heston model (1.1)-(4.1). Consequently, (4.4) is the density of the MEMM $Q_{M E}$ for the Heston model (4.1).

Proof. The proof is essentially the same as for (Benth and Karlsen [3], Proposition (4.2)]. We only have to clarify few points concerning the time-dependent parameters. Since $K_{t}=$ $\int_{0}^{t} \xi(s) Y_{s}^{2} d s$ and

$$
[L]_{t}=4 \int_{0}^{t} \beta^{2}(s)(1-\rho(s)) a^{2}(s) Y_{s}^{2} d s \leq 4 a^{2}(0) \beta^{2}(0)\left(1-\rho^{2}(0)\right) \int_{0}^{t} Y_{s}^{2} d s,
$$

we need to prove that $\sup _{n} \mathbb{E}_{Q_{n}}\left[\int_{0}^{T_{n}} Y_{s}^{2} d s\right]<\infty$ to verify Condition $A$. Recall that the process $\tilde{U}_{t}^{n}=\rho(t) \tilde{B}_{t \wedge T_{n}}+\sqrt{1-\rho^{2}(t)} \tilde{W}_{t \wedge T_{n}}$ is a Brownian motion under $Q_{n}$. Moreover, we find for $t \leq T_{n}$ that

$$
\begin{aligned}
d X_{t}= & \left(2 \lambda(t)+\beta^{2}(t)-(2 \alpha(t)+2 \beta(t) \xi(t) \rho(t)+\right. \\
& \left.\left.+4 \beta^{2}(t)\left(1-\rho^{2}(t)\right) a(t)\right) X_{t}\right) d t+2 \beta(t) \sqrt{X_{t}} d \tilde{U}_{t}^{2}
\end{aligned}
$$

Integrating from zero to $T_{n}$ yields,

$$
\begin{gathered}
\int_{0}^{T_{n}}\left(2 \alpha(t)+2 \beta(t) \xi(t) \rho(t)+4 \beta^{2}(t)\left(1-\rho^{2}(t)\right) a(t)\right) Y_{t}^{2} d t \\
\leq x+\int_{0}^{T}\left(2 \lambda(t)+\beta^{2}(t)\right) d t+2 \int_{0}^{T_{n}} \beta(t) Y_{t} d \tilde{U}_{t}^{n}
\end{gathered}
$$

since $X_{T_{n} \geq 0}$. Using the fact that $\tilde{U}_{t}^{n}$ is $Q_{n}$-Brownian, we find $\mathbb{E}_{Q_{n}}\left[\int_{0}^{T_{n}} Y_{t} d \tilde{U}_{t}^{n}\right]=0$ since

$$
\mathbb{E}_{Q_{n}}\left[\left(\int_{0}^{T_{n}} \sqrt{X_{t}} d \tilde{U}_{t}^{n}\right)^{2}\right]=\mathbb{E}_{Q_{n}}\left[\int_{0}^{T_{n}} Y_{t}^{2} d t\right]=\mathbb{E}_{Q_{n}}\left[K_{T_{n}}\right] \leq n .
$$

Hence we get

$$
\mathbb{E}_{Q_{n}}\left[\int_{0}^{T_{n}}\left(2 \alpha(t)+2 \beta(t) \xi(t) \rho(t)+4 \beta^{2}(t)\left(1-\rho^{2}(t)\right) a(t)\right) Y_{t}^{2} d t\right] \leq x+\int_{0}^{T}\left(2 \lambda(t)+\beta^{2}(t)\right) d t .
$$

From $a(t) \geq 0$, it holds that

$$
\left.2 \alpha(t)+2 \beta(t) \xi(t) \rho(t) \leq 2 \alpha(t)+2 \beta(t) \xi(t) \rho(t)+4 \beta^{2}(t)\left(1-\rho^{2}(t)\right) a(t)\right),
$$

and by assumption $\alpha(t)>\beta(t) \xi(t)$ and that functions $\lambda(t)$ and $\beta(t)$ are bounded; we find:

$$
\mathbb{E}_{Q_{n}}\left[\int_{0}^{T_{n}} Y_{t}^{2} d t\right] \leq \frac{x+\int_{0}^{T}\left(2 \lambda(t)+\beta^{2}(t)\right) d t}{2 \alpha(t)+2 \beta(t) \xi(t) \rho(t)}<\infty .
$$


Hence Condition $A$ holds.

To verify Condition $B$, we proceed as follows:

$$
\frac{\mu^{2}\left(t, Y_{t}\right)}{\sigma^{2}\left(t, Y_{t}\right)}+\delta\left(t, Y_{t}\right)=\xi^{2}(t) Y_{t}^{2}+\beta(t)\left(1-\rho^{2}(t)\right) 4 a^{2}(t) Y_{t}^{2} \leq k Y_{t}^{2}
$$

with $k=\xi^{2}(0)+4 \beta^{2}(0)\left(1-\rho^{2}(0)\right) a^{2}(0)$. Apply Hölder's inequality to reach:

$$
\mathbb{E}\left[\exp \left(\epsilon \int_{0}^{T} k Y_{t}^{2} d t\right)\right] \leq T^{-1} \sum_{n=0}^{\infty} \frac{(\epsilon k T)^{n}}{n !} \int_{0}^{T} \mathbb{E}\left[Y_{t}^{2 n}\right] d t .
$$

Note that $X_{t}=Y_{t}^{2}$ satisfies via Itô's formula the Cox-Ingersoll-Ross (CIR) process [4]

$$
d X_{t}=2 \alpha(t)\left(\frac{2 \lambda(t)+\beta^{2}(t)}{2 \alpha(t)}-X_{t}\right) d t+2 \beta(t) \sqrt{X_{t}} d U_{t}
$$

where

$$
d U_{t}=\rho(t) d B_{t}+\sqrt{1-\rho(t)} d W_{t}
$$

is a Brownian motion. From equation (4.6) we note that $Y_{t}^{2}$ is a CIR process which is non-centrally $\chi^{2}$-distributed. The probability density of $Y_{t}^{2}$ starting in $y^{2}$ at time $s$ is:

$$
f\left(z_{t}, t ; z_{s}, s\right)=c_{t} e^{-u-v}\left(\frac{u}{v}\right)^{\frac{q}{2}} I_{q}(2 \sqrt{u v}) .
$$

where $I_{q}$ is the modified Bessel function of the first kind of order $q$ and

$$
\begin{aligned}
c_{t} & \equiv \frac{4 \alpha(t)}{4 \beta^{2}(t)\left(1-e^{-2 \alpha(t)(t-s)}\right)}, \quad u \equiv c_{t} y^{2} e^{-2 \alpha(t)(t-s)}, \\
v & \equiv c_{t} z_{t}, \quad q \equiv \frac{2 \lambda(t)+\beta^{2}(t)}{2 \beta^{2}(t)}-1 .
\end{aligned}
$$

Rewriting slightly, we find that

$$
f(z ; t)=k_{t} c_{t} e^{-c_{t} z} z^{\frac{q}{2}} I_{q}\left(2 c_{t} y e^{-\alpha(t) t} \sqrt{z}\right),
$$

Here,

$$
k_{t}=e^{-c_{t} y^{2} e^{-2 \alpha(t)(t-s)}}\left(y^{2} e^{-2 \alpha(t)(t-s)}\right)^{\frac{-q}{2}},
$$

which can be bounded by a constant $C$ on $[s, T]$. Hence we find (using formula 9.6.18 on page 376 in Abramowitz and Stegun [1], see also Benth and Karlsen [3] and the FubiniTonelli theorem)

$$
\begin{aligned}
\mathbb{E}\left[X_{t}^{n}\right] & \leq C c_{t} \int_{0}^{\infty} z_{t}^{n+\frac{q}{2}} e^{c_{t} z_{t}} I_{q}\left(2 c_{t} y e^{-\alpha(t)(t-s)} \sqrt{z_{t}}\right) d z_{t} \\
& =C c_{t} \int_{0}^{\infty} z_{t}^{n+q} e^{-c_{t} z_{t}} \int_{0}^{\pi} \sin ^{2 q}(\theta) e^{2 c_{t} y e^{-\alpha(t)(t-s)} \cos (\theta) \sqrt{z_{t}}} d \theta d z_{t} \\
& =C c_{t} \int_{0}^{\pi} \int_{0}^{\infty} z_{t}^{n+q} e^{-c_{t} z_{t}+2 c_{t} y e^{-\alpha(t)(t-s)} \cos (\theta) \sqrt{z_{t}}} d \theta \sin ^{2 q}(\theta) d z_{t} \\
& =C c_{t} \int_{0}^{\infty} z_{t}^{n+q} e^{-c_{t} z_{t}+2 c_{t} y \sqrt{z_{t}}} d z_{t} .
\end{aligned}
$$


Here, $C$ has changed from line to line in the estimation process. By completion of the square we have

$$
e^{-c_{t} z_{t}+2 c_{t} y \sqrt{z_{t}}}=e^{c_{t}\left(\sqrt{z_{t}}-y\right)^{2}}
$$

where $e^{c_{t}\left(\sqrt{z_{t}}-y\right)^{2}}$ has a maximum point for $z_{t}=y^{2}$ and behaves like $e^{-c_{t} z_{t}}$ for $z_{t}$ large. We have:

$$
\begin{aligned}
\mathbb{E}\left[X_{t}^{n}\right] & \leq C c_{t} \int_{0}^{\infty} z_{t}^{n+q} e^{-c_{t} z_{t}} d z_{t} \\
& =C c_{t}^{-(n+q)} \Gamma(n+q+1) \\
& \leq C\left(\frac{\beta(t)^{2}}{\alpha(t)}\right)^{(n+q)} \Gamma(n+q+1) .
\end{aligned}
$$

Choosing $\epsilon$ sufficiently small gives:

$$
\mathbb{E}\left[\exp \left(\epsilon k \int_{0}^{T} Y_{t}^{2} d t\right)\right] \leq T^{-1} \sum_{n=0}^{\infty} \frac{(\epsilon k T)^{n}}{n !} \int_{0}^{T} \mathbb{E}\left[X_{t}^{n}\right] d t<\infty .
$$

Therefore, condition $B$ is fulfilled, and the proposition is proved.

Acknowledgment. The results of this paper were obtained during my Dphil. studies. I would like to express deep gratitude to my supervisor Prof. Fred Espen Benth whose guidance and support were crucial for the successful completion of this project.

\section{REFERENCES}

[1] M. Abramowitz and I. I. Stegun, (1972). Handbook of mathematical functions. Dover Publications: New York.

[2] F. Bellini and M. Frittelli, (2002). On the existence of minimax martingale measures. Mathematical Finance, 12(1), pp. 1-21.

[3] F. Benth and K. Karlsen, (2005). A PDE representation of the density of the minimum entropy martingale measure in stochastic volatility markets. Stochastics An International Journal of probability and Stochastic Processes, 77(2), pp. 109-123.

[4] J. C. Cox and J. E. Ingersoll., (1985). Theory of the term structure of interest rates. Econometrica, 53(2), pp. 385-407.

[5] F. Da Lio and O. Ley (2006). Uniqueness results for second order Bellman-Isaacs equations under quadratic growth assumptions and applications, SIAM Journal on Control and Optimisation 45(1), pp 74-106.

[6] F. Delbaen, P. Grandits, T. Rheinländer, D. Samperi, M. Schweizer and C. Stricker, (2002). Exponential hedging and entropic penalities. Mathematical Finance, 12(2), pp. 99-123.

[7] W. H. Fleming and H. M. Soner (1993). Controlled Markov and viscosity solutions. Springer-Verlag, New York.

[8] M. Frittelli (2000). The minimal entropy martingale and the valuation problem in incomplete markets. Mathematical Finance, 10(1), pp. 39-52.

[9] S. L. Heston (1993). A closed form solution for options with stochastic volatility with applications to bond and currency options. Review of Financial Studies, 6, pp. 327-343.

[10] D. Hobson (2004). Stochastic volatility models, correlation and the q-optimal measure. Mathematical Finance, 14(4), pp. 537-556. 
[11] B. Øksendal (1998). Stochastic Differential Equations. An Introduction with Applications Fifth Edition. Springer.

[12] H. Pham, (2000). On quadratic hedging in continuous time. Mathematical Methods of Operational Research, 51(2), pp 315-339

[13] P. Grandits and T. Rheinländer (2002). On the minimal entropy martingale measure. Annals of Probability., 30(3), pp. 1003-1038.

[14] S. D. Hodges and A. Neuberger (1989). Optimal Replication of Contingent Claims Under Transaction Costs. Reviews of Future Markets., 8, pp. 222-239.

[15] N. Ikeda and S. Watanabe, (1981). Stochastic differential equations and diffusion processes. Amsterdam : North-Holland, Kodansha.

[16] T. Rheinländer, Preprint, (2003). An entropy approach to the Stein/ Stein model with correlation. ETH Zürich.

[17] M. Mania, M. Santacrose and T. Tevzadze, (2003). A semimartingale BSDE related to the minimal entropy martingale measure. Finance and Stochastics., 7, pp. 385-402.

[18] W. Schachermayer, (2001). Optimal investment in incomplete markets when wealth may become negative. Annals of Applied Probability, 11(3), pp. 694-734.

Department of Mathematics and Applied Mathematics, University of Pretoria, 0002, South Africa

E-mail address: rodwell.kufakunesu@up.ac.za 